\title{
A variant of hybrid conjugate gradient methods based on the convex combination for optimization
}

\author{
Basim A. Hassan ${ }^{1}$, Ahmed O. Owaid ${ }^{2}$, Zena T. Yasen ${ }^{3}$ \\ ${ }^{1,3}$ Department of Mathematics,College of Computers Sciences and Mathematics, University of Mosul, Iraq \\ ${ }^{2}$ Department of studies and planning, Presidency of Mosul University, University of Mosul, Iraq
}

\section{Article Info}

\section{Article history:}

Received Oct 12, 2019

Revised Apr 27, 2020

Accepted Jul 13, 2020

\section{Keywords:}

Descent property

Global convergence property

Hybeid conjugate gradient

\begin{abstract}
On some studies a conjugate parameter plays an important role for the conjugate gradient methods. In this paper, a variant of hybrid is provided in the search direction based on the convex combination. This search direction ensures that the descent condition holds. The global convergence of the variant of hybrid is also obtained. Our strong evidence is a numerical analysis showing that the proposed variant of hybrid method is efficient than the Hestenes and Stiefel method.
\end{abstract}

\section{Corresponding Author:}

Basim A. Hassan,

Department of Mathematics,

College of Computers Sciences and Mathematics,

University of Mosul, Iraq.

Email: basimah@uomosul.edu.iq, basimabas39@gmail.com

\section{INTRODUCTION}

The conjugate gradient [1] is an adaptation of the optimization method to large-scale problems. Here, we matter with the following problem:

$$
\operatorname{Min} f(x), \mathrm{x} \in \mathrm{R}^{\mathrm{n}}
$$

to denote the objective function, and $g(x)$, denote the gradient $f(x)$, respectively.

Many conjugate gradient methods for (1) produce a sequence $\left\{x_{k}\right\}$ by the recurrence:

$$
x_{0} \in R^{n}, x_{k+1}=x_{k}+\alpha_{k} d_{k}
$$

where $d_{k}$ is a search direction and $\alpha_{k}$ is a step size which was calculated by applying line search rules, the most important and famous is the Wolfe conditions:

$$
\begin{aligned}
& f\left(x_{k}+\alpha_{k} d_{k}\right) \leq f\left(x_{k}\right)+\delta \alpha_{k} g_{k}^{T} d_{k} \\
& d_{k}^{T} g\left(x_{k}+\alpha_{k} d_{k}\right) \geq \sigma d_{k}^{T} g_{k}
\end{aligned}
$$


where $0<\delta<\sigma$. For details see [2].

Therefore, this study will focus on the CG method whose direction of search is written as:

$$
d_{k+1}=-g_{k+1}+\beta_{k} d_{k}
$$

where $\beta_{k}$ is a scalar known as the CG update. The suitable choice for $\beta_{k}$ leads to improve numerical performance conjugate gradient methods. There are some well known and effective conjugate gradient methods, such as, [3-6].

The two most popular formulas know in conjugate gradient methods are as follows:

$$
\beta_{k}^{D Y}=\frac{\left\|g_{k+1}\right\|^{2}}{d_{k}^{T} y_{k}}, \quad \beta_{k}^{H S}=\frac{g_{k+1}^{T} y_{k}}{d_{k}^{T} y_{k}}
$$

where $y_{k}=g_{k+1}-g_{k}$. The first is better in terms of convergence and the second is better in numerical performance. More details can be found in [7, 8]. is given by:

Yuan [9], claimed that the parameter conjugate gradient method has best numerical performance

$$
\beta_{k}^{Y}=\frac{g_{k+1}^{T} y_{k}}{\left(f\left(x_{k}\right)-f\left(x_{k+1}\right)\right) / \alpha_{k}-g_{k}^{T} d_{k} / 2}
$$

Newly, in [10], presented a modified Yuan method has good convergence property is given by:

$$
\beta_{k}^{B}=\frac{g_{k+1}^{T} g_{k+1}}{\left(f\left(x_{k}\right)-f\left(x_{k+1}\right)\right) / \alpha_{k}-g_{k}^{T} d_{k} / 2} .
$$

The global convergence property is an important property of conjugate gradient methods, and has as many results as we can see in $[1,11]$.

The urge of this paper is to get great algorithms we combine the benefits of the two formulas $\beta_{k}^{Y}$ and $\beta_{k}^{B}$. Provide novel algorithms have fantastic numerical performance with good better convergence and a discussion for the theoretical properties and the numerical results.

\section{A CONVEX COMBINATION OF $\beta_{k}^{B}$ AND $\beta_{k}^{Y}$ METHODS}

In vision of the gentle convergence property of the $\beta_{k}^{B}$ method, and the efficient performance of the $\beta_{k}^{Y}$ method, we paying attention on designing new methods which possess the above properties simultaneously. The parameter $\beta_{k}$ of the hybrid conjugate gradient method of $\beta_{k}^{B}$ and $\beta_{k}^{Y}$ is formulized as:

$$
\beta_{k}^{H Y B}=\left(1-\vartheta_{k}\right) \beta_{k}^{Y}+\vartheta_{k} \beta_{k}^{B}
$$

So, we get:

$$
d_{k+1}^{H Y B}=-g_{k+1}+\beta_{k}^{H Y B} s_{k}, \quad s_{k}=x_{k+1}-x_{k}
$$

To be determined the scalar parameter $\vartheta_{k}$, the see it : if $\vartheta_{k}=0$, then $\beta_{k}^{H Y B}=\beta_{k}^{Y}$ and $\vartheta_{k}=1$, then $\beta_{k}^{H Y B}=\beta_{k}^{B}$. If $0<\vartheta_{k}<1$, then $\beta_{k}^{H Y B}$ is a proper convex combination of the parameters is $\beta_{k}^{Y}$ and $\beta_{k}^{B}$. 
Theorem 2.1:

If the relationships (9) and (10) holds, then:

$$
d_{k+1}^{H Y B}=\left(1-\vartheta_{k}\right) d_{k+1}^{Y}+\vartheta_{k} d_{k+1}^{B}
$$

Proof :

Possessing perspective relationships $\beta_{k}^{B}$ and $\beta_{k}^{Y}$, the relationship (9) becomes:

$$
\beta_{k}^{H Y B}=\frac{\left(1-\vartheta_{k}\right) g_{k+1}^{T} y_{k}+\vartheta_{k} g_{k+1}^{T} g_{k+1}}{\left(f\left(x_{k}\right)-f\left(x_{k+1}\right)\right) / \alpha_{k}-g_{k}^{T} d_{k} / 2}
$$

So, the relation (10) becomes:

$$
d_{0}^{H Y B}=-g_{0}, d_{k+1}^{H Y B}=-g_{k+1}+\frac{\left(1-\vartheta_{k}\right) g_{k+1}^{T} y_{k}+\vartheta_{k} g_{k+1}^{T} g_{k+1}}{\left(f\left(x_{k}\right)-f\left(x_{k+1}\right)\right) / \alpha_{k}-g_{k}^{T} d_{k} / 2} s_{k}
$$

In the other mind of a relationship (13) we have:

$$
d_{k+1}^{H Y B}=-\left(\vartheta_{k} g_{k+1}+\left(1-\vartheta_{k}\right) g_{k+1}\right)+\beta_{k}^{H Y B} s_{k},
$$

Implies that:

$$
d_{k+1}^{H Y B}=-\left(\vartheta_{k} g_{k+1}+\left(1-\vartheta_{k}\right) g_{k+1}\right)+\left(\left(1-\vartheta_{k}\right) \beta_{k}^{Y}+\vartheta_{k} \beta_{k}^{B}\right) s_{k}
$$

The last relation yields:

$$
d_{k+1}^{H Y B}=\vartheta_{k}\left(-g_{k+1}+\beta_{k}^{B} s_{k}\right)+\left(1-\vartheta_{k}\right)\left(-g_{k+1}+\beta_{k}^{Y} s_{k}\right)
$$

From (16) we finally conclude:

$$
d_{k+1}^{H Y B}=\left(1-\vartheta_{k}\right) d_{k+1}^{Y}+\vartheta_{k} d_{k+1}^{B}
$$

Apply conjugacy condition to find value $\vartheta_{k}$ in our method:

$$
y_{k}^{T} d_{k}^{H Y B}=0
$$

Multiplying (13) by $y_{k}^{T}$ and applying (18) we get:

$$
\begin{aligned}
& y_{k}^{T}\left[-g_{k+1}+\frac{\left(1-\vartheta_{k}\right) g_{k+1}^{T} y_{k}+\vartheta_{k} g_{k+1}^{T} g_{k+1}}{\left(f\left(x_{k}\right)-f\left(x_{k+1}\right)\right) / \alpha_{k}-g_{k}^{T} d_{k} / 2} s_{k}\right]=0 \\
& -y_{k}^{T} g_{k+1}+\frac{\left(1-\vartheta_{k}\right) g_{k+1}^{T} y_{k}\left(y_{k}^{T} s_{k}\right)+\vartheta_{k} g_{k+1}^{T} g_{k+1}\left(y_{k}^{T} s_{k}\right)}{\left(f\left(x_{k}\right)-f\left(x_{k+1}\right)\right) / \alpha_{k}-g_{k}^{T} d_{k} / 2}=0,
\end{aligned}
$$

So,

$$
y_{k}^{T} g_{k+1}-\frac{g_{k+1}^{T} y_{k}}{\left(f\left(x_{k}\right)-f\left(x_{k+1}\right)\right) / \alpha_{k}-g_{k}^{T} d_{k} / 2}\left(y_{k}^{T} s_{k}\right)=\vartheta_{k}\left[\frac{g_{k+1}^{T} g_{k+1}\left(y_{k}^{T} s_{k}\right)-g_{k+1}^{T} y_{k}\left(y_{k}^{T} s_{k}\right)}{\left(f\left(x_{k}\right)-f\left(x_{k+1}\right)\right) / \alpha_{k}-g_{k}^{T} d_{k} / 2}\right],
$$


i.e.

$$
\frac{\left(\left(f\left(x_{k}\right)-f\left(x_{k+1}\right)\right) / \alpha_{k}-g_{k}^{T} d_{k} / 2-y_{k}^{T} s_{k}\right)}{\left(f\left(x_{k}\right)-f\left(x_{k+1}\right)\right) / \alpha_{k}-g_{k}^{T} d_{k} / 2}\left(y_{k}^{T} g_{k+1}\right)=\vartheta_{k} \frac{g_{k+1}^{T} g_{k}}{\left(f\left(x_{k}\right)-f\left(x_{k+1}\right)\right) / \alpha_{k}-g_{k}^{T} d_{k} / 2}\left(y_{k}^{T} s_{k}\right)
$$

Finally,

$$
\vartheta_{k}=\frac{\left(\left(f\left(x_{k}\right)-f\left(x_{k+1}\right)\right) / \alpha_{k}-g_{k}^{T} d_{k} / 2-y_{k}^{T} s_{k}\right)\left(y_{k}^{T} g_{k+1}\right)}{g_{k+1}^{T} g_{k}\left(y_{k}^{T} s_{k}\right)}
$$

The appropriate value for $\vartheta_{k}$, is in the interval $[0,1]$. We get $\beta_{k}^{H Y B}=\beta_{k}^{Y}$ if $\vartheta_{k} \leq 0$, then set $\vartheta_{k}=0$ in (9), and we get $\beta_{k}^{H Y B}=\beta_{k}^{B}$ if $\vartheta_{k} \geq 1$, then set $\vartheta_{k}=1$ in (9). If it's a value of $\vartheta_{k}$ is specified, $\beta_{k}^{H Y B}$ it is combines the properties of the $\mathrm{Y}$ and the $\mathrm{B}$ algorithms in a convex way.

Now we formally state our algorithm and call by HYB:

\section{Algorithm (HYB):}

Stage 1. Initialization : $x_{1} \in R^{n}, 0<\delta_{1}<\delta_{2}<1, d_{1}=-g_{1}$ and $\alpha_{1}=1 /\left\|g_{1}\right\|$.

Stage 2. If $\left\|g_{k+1}\right\| \leq 10^{-6}$, then stop.

Stage 3. Compute: $\alpha_{k}$ by using the Wolfe condition and let $x_{k+1}=x_{k}+\alpha_{k} d_{k}$.

Stage 4. Computation $\vartheta_{k}$, if $g_{k+1}^{T} g_{k}\left(y_{k}^{T} s_{k}\right)=0$, then set $\vartheta_{k}=0$, else set $\vartheta_{k}$ as in (23) respectively.

Stage 5. Compute : $\beta_{k}^{H Y B}$ as in (9).

Stage 6. Compute : $d_{k+1}=-g_{k+1}+\beta_{k} s_{k}$. Put $k=k+1$ go to step 2 .

\section{CONVERGENCE ANALYSIS}

We adopt the following assumption used often in the literature to analyze the global convergence.

\section{Assumption 3.1:}

suppose that the gradient is "Lipschitz continuous". Then for some positive constant $L$, we have:

$$
\|g(n)-g(m)\| \leq L\|n-m\|, \quad \forall n, m \in U \text {. }
$$

where we assume the level set:

$$
S=\left\{x \in R^{n} \mid f(x) \leq f\left(x_{0}\right)\right\}
$$

is bounded set. Under these assumptions of $f(x)$, there exists a constant $\Gamma \geq 0$ such that:

$$
\left\|g_{k+1}\right\| \leq \Psi
$$

More details can be found in $[12,13]$.

Now, we will focus the proof of the descent property.

\section{Theorem (3.1)}

Assume that (24) and (26) hold and let Wolfe conditions hold. Also, let $\left\{\left\|s_{k}\right\|\right\}$ tend to zero, and consider there exist two positive constants $\xi_{1}, \xi_{2}$ we satisfies:

$$
\left(f\left(x_{k}\right)-f\left(x_{k+1}\right)\right) / \alpha_{k}-g_{k}^{T} d_{k} / 2 \geq \xi_{1}\left\|s_{k}\right\|^{2},
$$




$$
\left\|g_{k+1}\right\|^{2} \leq \xi_{2}\left\|s_{k}\right\|
$$

then $d_{k}^{H Y B}$ satisfies the $g_{k+1}^{T} d_{k+1}^{H Y B} \leq 0$, for all $k$.

\section{Proof :}

It fists $d_{0}=-g_{0}$. If $k=0$, it holds $g_{0}^{T} d_{0}=-\left\|g_{0}\right\|^{2}<0$. Multiplying (11) by $g_{k+1}^{T}$, we obtained:

$$
g_{k+1}^{T} d_{k+1}^{H Y B}=\left(1-\vartheta_{k}\right) g_{k+1}^{T} d_{k+1}^{Y}+\vartheta_{k} g_{k+1}^{T} d_{k+1}^{B}
$$

If $\vartheta_{k}=0$, the relation (29) becomes:

$$
g_{k+1}^{T} d_{k+1}^{H Y B}=g_{k+1}^{T} d_{k+1}^{Y}
$$

So, if $\vartheta_{k}=0$, the hybrid method satisfies the descent condition, if it fists for Y method. By using the conditions of Theorem 3.1, we can prove the descent for Y method. It fists:

$$
d_{k+1}^{Y}=-g_{k+1}+\beta_{k}^{Y} s_{k}
$$

Multiplying (31) by $g_{k+1}^{T}$, we get:

$$
g_{k+1}^{T} d_{k+1}^{Y}=-g_{k+1}^{T} g_{k+1}+\beta_{k}^{Y} g_{k+1}^{T} s_{k}
$$

Using definition $\beta_{k}^{Y}$, we get:

$$
g_{k+1}^{T} d_{k+1}^{Y}=-g_{k+1}^{T} g_{k+1}+\frac{g_{k+1}^{T} y_{k}}{\left(f\left(x_{k}\right)-f\left(x_{k+1}\right)\right) / \alpha_{k}-g_{k}^{T} d_{k} / 2} g_{k+1}^{T} s_{k}
$$

From (33) we get:

$$
g_{k+1}^{T} d_{k+1}^{Y} \leq-\left\|g_{k+1}\right\|^{2}+\frac{\left\|g_{k+1}\right\|^{2}\left\|y_{k}\right\|\left\|s_{k}\right\|}{\left(f\left(x_{k}\right)-f\left(x_{k+1}\right)\right) / \alpha_{k}-g_{k}^{T} d_{k} / 2}
$$

From Lipschitz condition we have $\left\|y_{k}\right\| \leq L\left\|s_{k}\right\|$, so:

$$
g_{k+1}^{T} d_{k+1}^{Y} \leq-\left\|g_{k+1}\right\|^{2}+\frac{\left\|g_{k+1}\right\|^{2} L\left\|s_{k}\right\|}{\left(f\left(x_{k}\right)-f\left(x_{k+1}\right)\right) / \alpha_{k}-g_{k}^{T} d_{k} / 2}
$$

But, using (27)-(28) we get:

$$
g_{k+1}^{T} d_{k+1}^{Y} \leq-\left\|g_{k+1}\right\|^{2}+\frac{\xi_{2} L\left\|s_{k}\right\|}{\xi_{1}}
$$

But, benefit from $\left\|s_{k}\right\| \Rightarrow 0$, the second part in (36) tends to zero, so there exists a $0<\delta \leq 1$, such that:

$$
\frac{1}{\xi_{1}} \xi_{2} L\left\|s_{k}\right\| \leq \delta\left\|g_{k+1}\right\|^{2}
$$


Now, from (36) we have:

$$
g_{k+1}^{T} d_{k+1}^{Y} \leq-\left\|g_{k+1}\right\|^{2}+\delta\left\|g_{k+1}\right\|^{2},
$$

i.e.

$$
g_{k+1}^{T} d_{k+1}^{Y} \leq-(1-\delta)\left\|g_{k+1}\right\|^{2}<0
$$

If either $\vartheta_{k}=1$, the relationship (29) we have:

$$
g_{k+1}^{T} d_{k+1}^{H Y B}=g_{k+1}^{T} d_{k+1}^{B}
$$

But, the descent property of B-method is proved in [10], by using Wolfe conditions.

Now, let $0<\vartheta_{k}<1$ and from (29), we get:

$$
g_{k+1}^{T} d_{k+1}^{H Y B} \leq\left(1-\vartheta_{k}\right) g_{k+1}^{T} d_{k+1}^{Y}+\vartheta_{k} g_{k+1}^{T} d_{k+1}^{B}
$$

We obviously can conclude now:

$$
g_{k+1}^{T} d_{k+1}^{H Y B} \leq 0
$$

We adopt the next theorem used commonly in the research literatures.

\section{Theorem (3.2)}

Let the iterative method of the form (2) and (5), where $d_{k}$ satisfies a $g_{k}^{T} d_{k}<0$ and $\alpha_{k}$ satisfies strong Wolfe conditions. If (24) holds, then either,

$$
\liminf _{k \rightarrow \infty}\left\|g_{k}\right\|=0
$$

Or,

$$
\sum_{k \geq 1} \frac{\left\|g_{k+1}\right\|^{4}}{\left\|d_{k+1}\right\|^{2}}<\infty
$$

It was originally given by Zoutendijk [14].

\section{Theorem (3.3)}

Let conditions of Theorem 3.2 holds. Then either $g_{k}=0$ for some $k$, or,

$$
\lim _{k \rightarrow \infty} \inf \left\|g_{k}\right\|=0
$$

\section{Proof :}

Let $g_{k} \neq 0, \forall k$. Using, a contrary to prove (45), that there exists a number $c>0$, such that:

$$
g_{k+1} \geq c, \forall k
$$


From (11) we get:

$$
\left\|d_{k+1}^{H Y B}\right\| \leq\left\|d_{k+1}^{Y}\right\|+\left\|d_{k+1}^{B}\right\|
$$

Next, it holds:

$$
\left\|d_{k+1}^{B}\right\| \leq\left\|g_{k+1}\right\|+\left|\beta_{k}^{B}\right|\left\|s_{k}\right\|
$$

From (9), (26)-(28), and (48) we get:

$$
\left\|d_{k+1}^{B}\right\| \leq \Psi+\frac{\xi_{2}}{\xi_{1}}
$$

Also,

$$
\left\|d_{k+1}^{Y}\right\| \leq\left\|g_{k+1}\right\|+\left|\beta_{k}^{Y}\right|\left\|s_{k}\right\|
$$

Using (26)-(28)and (50) we get:

$$
\left\|d_{k+1}^{Y}\right\| \leq \Psi+\frac{\Psi L}{\xi_{1}}
$$

So, using (47), (49) and (51) we get:

$$
\left\|d_{k+1}^{H Y B}\right\| \leq 2 \Psi+\frac{\Psi L}{\xi_{1}}+\frac{\xi_{2}}{\xi_{1}}
$$

We obtained:

$$
\frac{\left\|g_{k+1}\right\|^{4}}{\left\|d_{k+1}\right\|^{2}} \geq \frac{c^{4}}{\left[2 \Psi+\frac{\Psi L}{\xi_{1}}+\frac{\xi_{2}}{\xi_{1}}\right]^{2}}
$$

where from:

$$
\sum_{k \geq 1}^{\infty} \frac{\left\|g_{k+1}\right\|^{4}}{\left\|d_{k+1}\right\|^{2}}=\infty
$$

Applying the Theorem 3.2, we obtain a contradiction. The proof is finish.

\section{NUMERICAL RESULTS AND DISCUSSION}

In this section, we report some numerical results with the proposed method and HS-method. We test the performance of Algorithm 2 on the following (15) problems with various sizes. Using Fortran 90 to encrypt these methods. In our application, we choose the following parameters : $\delta_{1}=0.001$ and $\delta_{2}=0.9$ Are selected examination problems of references [15]. Stop state is $\left\|g_{k+1}\right\| \leq 10^{-6}$.

It has been reported numerical results in Table 1. Represents the first column and the name of the second problem and its dimensions in [15], respectively. Other test functions have been used in various

A Variant of Hybrid Conjugate Gradient Methods Based on the Convex Combination... (Basim A. Hassan) 
researches such as [16-25] "NI and NR and NF in the table indicates the number of iterations and the number of evaluations of jobs and the number of replay restart, respectively". Relativa efficiency of the variant of hybrid Algorithm as shown in Table 2.

Table 1. Comparing different conjugate gradient methods with different test functions

\begin{tabular}{|c|c|c|c|c|c|c|c|}
\hline & & \multicolumn{3}{|c|}{ HS algorithm } & \multicolumn{3}{|c|}{ HYB algorithm } \\
\hline P. No. & $\mathrm{n}$ & NI & NR & NF & NI & NR & NF \\
\hline \multirow[t]{2}{*}{ Trigonometric } & 100 & 19 & 10 & 35 & 20 & 11 & 37 \\
\hline & 1000 & 39 & 22 & 67 & 34 & 22 & 63 \\
\hline \multirow[t]{2}{*}{ Perturbed Quadratic } & 100 & 102 & 33 & 155 & 93 & 26 & 148 \\
\hline & 1000 & 326 & 91 & 509 & 317 & 92 & 509 \\
\hline \multirow[t]{2}{*}{ Extended PSC1 } & 100 & 8 & 6 & 17 & 8 & 6 & 17 \\
\hline & 1000 & 26 & 25 & 505 & 7 & 5 & 15 \\
\hline \multirow[t]{2}{*}{ Q. Diagonal Perturbed } & 100 & 47 & 6 & 84 & 53 & 9 & 95 \\
\hline & 1000 & 188 & 29 & 335 & 173 & 24 & 309 \\
\hline \multirow[t]{2}{*}{ Extended Wood } & 100 & 32 & 13 & 60 & 34 & 11 & 64 \\
\hline & 1000 & 28 & 11 & 54 & 28 & 9 & 49 \\
\hline \multirow[t]{2}{*}{ ARWHEAD (CUTE) } & 100 & 12 & 7 & 23 & 8 & 5 & 16 \\
\hline & 1000 & 17 & 11 & 148 & 8 & 6 & 56 \\
\hline \multirow[t]{2}{*}{ NONDIA (CUTE) } & 100 & 17 & 9 & 34 & 13 & 7 & 26 \\
\hline & 1000 & 12 & 7 & 25 & 13 & 7 & 27 \\
\hline \multirow[t]{2}{*}{ Partial Perturbed Q. } & 100 & 82 & 23 & 124 & 76 & 21 & 118 \\
\hline & 1000 & 260 & 57 & 429 & 259 & 65 & 429 \\
\hline \multirow[t]{2}{*}{ LIARWHD (CUTE) } & 100 & 21 & 12 & 40 & 19 & 11 & 34 \\
\hline & 1000 & 20 & 12 & 45 & 19 & 11 & 44 \\
\hline \multirow[t]{2}{*}{ DENSCHNF (CUTE) } & 100 & 20 & 17 & 36 & 18 & 17 & 32 \\
\hline & 1000 & 22 & 17 & 41 & 18 & 16 & 33 \\
\hline \multirow{2}{*}{ SINCOS } & 100 & 8 & 6 & 17 & 8 & 6 & 17 \\
\hline & 1000 & 26 & 25 & 505 & 7 & 5 & 15 \\
\hline \multirow[t]{2}{*}{ Generalized quartic GQ2 } & 100 & 34 & 10 & 55 & 31 & 7 & 54 \\
\hline & 1000 & 38 & 12 & 63 & 36 & 9 & 63 \\
\hline \multirow[t]{2}{*}{ Diagonal 2} & 100 & 61 & 18 & 103 & 66 & 26 & 105 \\
\hline & 1000 & 192 & 57 & 321 & 180 & 63 & 303 \\
\hline \multirow[t]{2}{*}{ Extended Three Expo Terms } & 100 & 13 & 7 & 20 & 8 & 5 & 13 \\
\hline & 1000 & 26 & 19 & 260 & 28 & 21 & 365 \\
\hline \multirow{2}{*}{ Quadratic QF1 } & 100 & 100 & 29 & 152 & 95 & 31 & 144 \\
\hline & 1000 & 371 & 105 & 586 & 375 & 110 & 604 \\
\hline Total & & 2095 & 684 & 4730 & 1985 & 648 & 3687 \\
\hline
\end{tabular}

From Table 1, it is completed that the more efficient algorithm in terms of the number of iterations is our method, being the best for $6 \%$ of the problems, followed by number of function evaluations, that was the efficient for nearly $22 \%$ of the problems and $6 \%$ in terms of the number of restart calls .

Table 2. Relativa efficiency of the variant of hybrid algorithm

\begin{tabular}{cccc}
\hline & NI & NR & NF \\
\hline HS algorithm & $100 \%$ & $100 \%$ & $100 \%$ \\
HYB algorithm & $94.74 \%$ & $94.73 \%$ & $77.94 \%$ \\
\hline
\end{tabular}

\section{CONCLUSIONS}

The variant of hybrid of the conjugate gradient direction is based on the convex combination. The search direction in the variant of hybrid satisfies as a descent condition and the global convergence is also obtained. Show that variant of hybrid method are the best for solving unconstrained optimization problems.

\section{ACKNOWLEDGMENT}

The authors are very grateful to the University of Mosul / College of Computers Sciences and Mathematics for their provided facilities, which helped to improve the quality of this work.

\section{REFERENCES}

[1] Hager W. W. and Zhang. H, "A surrevy of nonlinear conjugate gradient methods," Paaific Journal of optimization, vol. 2, pp.35-58, 2006.

[2] Zhongbo S., Hongyang L., Jing W. and Yantao T. "Two modified spectral conjugate gradient methods and their global convergence for unconstrained optimization." International Journal of Computer Mathematics, to be apper, 2017.

[3] Fletcher, R. and Reeves C. "Function minimization by conjugate gradients," Computer J, vol. 7, pp. 149-154, 1964.

[4] Fletcher R., "Practical Method of Optimization (2nd Edition)," John Wiley and Sons, New York, 1989. 
[5] Liu Y. and Storey C. "Efficient generalized conjugate gradients algorithms," Part 1: Theory. J. Optimization Theory and Applications, vol. 69, pp. 129-137, 1991.

[6] Polak, E. and Ribiere, G. "Note for Convergence Direction Conjugate," Revue Francaise Informant, Reserche. Opertionelle, pp. 35-43. 1969.

[7] Dai Y. H. and Yuan Y., "A nonlinear conjugate gradient method with a strong global convergence property," SIAM J. optimization, pp. 177-182, 1999.

[8] Hestenes, M. R. and Stiefel, E. L. "Method of conjugate gradients for Solving linear systems," Journal National Standards, vol. 49, pp. 409-436, 1952.

[9] Yuan Y., "Some problems in nonlinear programming, in Yuan," Y., (Ed.) Numerical Linear Algebra and Optimization, Science Press, Beijing / New York, pp.90-110, 2003.

[10] Basim A. Hassan, "A new formula for conjugate parameter computation based on the quadratic model," Indonesian Journal of Electrical Engineering and Computer Science (IJEECS), vol. 3, no. 3, pp. 954-961, 2019.

[11] Sun, W., Yuan, Y., "Optimization Theory and Methods: Nonlinear Programming," Springer, New York, 2006.

[12] Hager W.W. and Zhang H., "A new conjugate gradient method with guaranteed descent and an efficient line search," SIAM J. Optim. vol. 16, pp. 170-192, 2005.

[13] Narushima Y. and Yabe H., "A survey of sufficient descent conjugate gradient methods for unconstrained optimization," SUT J. Math. 50, pp. 167-203, 2014.

[14] Zoutendijk, G., "Nonlinear programming, computational methods," In: Abadie, J. (eds.) Integerand Nonlinear Programming, North-Holland, Amsterdam, pp. 37-86, 1970.

[15] Andrie N. "An Unconstrained Optimization Test functions collection," Advanced Modeling and optimization, vol. 10, pp. 147-161, 2008.

[16] Andrei N., "Test functions for unconstrained optimization," Research Institute for informatics, Center for Advanced Modeling and Optimization, pp. 1-15, 2004.

[17] Djordjevic S. S., "New Hybrid Conjugate Gradient Method as a Convex Combination of LS and CD Methods," Filomat, vol. 31, no. 6, pp. 1813-1825, 2017.

[18] Bongartz I., et al., "CUTE: Constrained and Unconstrained Testing Environment," ACM Transactions on Mathematical Software, vol. 21, no. 1, pp. 123-160, 1995.

[19] Alhawarat A., et al., "An efficient hybrid conjugate gradient method with the strong Wolfe-Powell line search," Mathematical Problems in Engineering, vol. 2015, no. 14, pp. 1-7, 2015.

[20] Basim A. H. , Zeyad M. A. and Hawraz N. J. "A descent extension of the Dai - Yuan conjugate gradient technique," Indonesian Journal of Electrical Engineering and Computer Science, pp. 661-668, 2019.

[21] Basim A. H. Hussein O. D.and Azzam S. Y. "A new kind of parameter conjugate gradient for unconstrained optimization," Indonesian Journal of Electrical Engineering and Computer Science, pp. 404-411, 2020.

[22] Basim A. Hassan, Osama M.T. W. and Ayad A. M. "A Class of Descent Conjugate Gradient Methods for Solving Optimization Problems," HIKARI Ltd, Applied Mathematical Sciences, pp. 12, 559 - 567, 2019.

[23] Basim A. Hassan and Mohammed W. T. "A Modified Quasi-Newton Equation in the Quasi-Newton Methods for Optimization”, HIKARI Ltd, Applied Mathematical Sciences, pp. 463 - 472, 2019.

[24] Basim A. Hassan, "A Globally Convergence Spectral Conjugate Gradient Method for Solving Unconstrained Optimization Problems and Mohammed," Raf. J. of Comp. \& Math's., pp. 21-28, 2013.

[25] Liu J.K.and Li S. J.,"New hybrid conjugate gradient method for unconstrained optimization,” Applied Mathematics and Computation, vol. 245, pp. 36-43, 2014. 